maximum entstehen; ob ein solches wirklich entsteht, hängt natürlich von der Verteilung der Teilchen selbst ab. Die Querschinitte der Ringe müssen ebenfalls von der Verteilung der Knotenlinien abhängen; sind dieselben in einer Richtung zusammengedrängt, so erscheint der betreffende Ring in dieser Hörsholm, I9I 7 Juli.
Richtung weit flacher als in der Richtung senkrecht dazu, sodaß die Schattenanomalien nur in bestimmten Stellungen des Planeten auftreten können. Werden die Knoten wieder durch die Störungen zerstreut, so können die Schattenanomalien in allen möglichen Stellungen des Planeten auftreten.

\title{
Über die Sichtbarkeit der Marskanäle in der Opposition 1913-14.
}

In seinen Monthly Reports on Mars (Pop. Astr., Jan.Sept. I 9 I 4) gibt Prof. $W$. H. Pickering mehrere Verzeichnisse der in der Opposition I9 I $^{-1} 4$ gesehenen "Kanäle ", die eine Prüfung meiner Ergebnisse in A. N. 200.50 gestatten.

Werden nur die in mindestens drei Nächten gesehenen Streifen in Betracht gezogen, so bekommt man aus Pickerings Beobachtungen die folgenden Mittelwerte für die erste $\left(T_{1}\right)$ bezw. letzte $\left(T_{2}\right)$ Beobachtung der betreffenden Streifen.

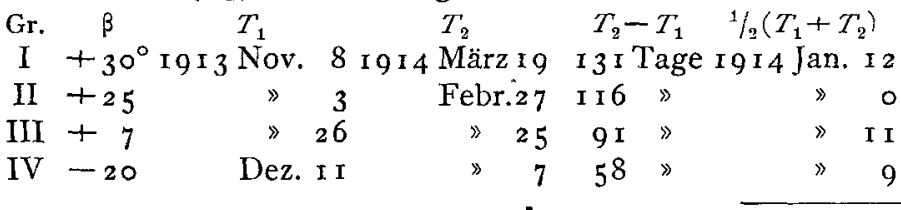

\section{Zur Reduktion der Beobachtungen am Passagenrohr.}

Die Grundformel für diese Reduktion ist die bekannte Mayersche Formel:

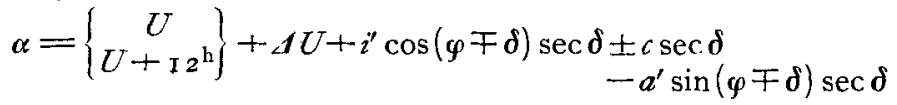

welche es erlaubt, die drei Instrumentalkonstanten, Neigung $i^{\prime}$, Kollimation $c$ und Azimut $a^{\prime}$ (welches aber hier positiv nach $\mathrm{N}$ gezählt wird), sowie den Uhrstand aus geeigneten Beobachtungen herzuleiten. Die Koeffizienten sind in den meisten astronomischgeodätischen und nautischen Tafeln tabuliert. Die Entnahme wird aber durch die doppelte Interpolation manchmal unsicher. Es läßt sich nun eine Transformation finden, durch die man die Koeffizienten der neuen Formel auf sehr einfache Weise sicher bilden kann. Es ist nämlich:

$$
\begin{aligned}
i^{\prime} \cos (\varphi \mp \delta) \sec \delta & =i^{\prime}(\cos \varphi \pm \sin \varphi \operatorname{tg} \delta) \\
& =i^{\prime} \sin \varphi(\operatorname{ctg} \varphi \pm \operatorname{tg} \delta) \\
-a^{\prime} \sin (\varphi \mp \delta) \sec \delta & =-a^{\prime}(\sin \varphi \mp \cos \varphi \operatorname{tg} \delta) \\
& =a^{\prime} \cos \varphi( \pm \operatorname{tg} \delta-\operatorname{tg} \varphi) .
\end{aligned}
$$

Setzt man nun $i^{\prime} \sin \varphi=i, \quad a^{\prime} \cos \varphi=a$
Die Gruppe I umfaßt Hades und Hyblaeus, Gruppe II Erebus, Eunostus, Styx und Nilokeras, Gruppe III Achelous, Cerberus, Thoth, Orontes, Pactolus, Nepenthes, Tartarus, Triton, Brontes und Jamuna, und Gruppe IV endlich Nectar, Daemon und Agathodaemon.

Die starke Abnahme der Sichtbarkeitsdauer $T_{2}-T_{1}$ mit wachsender Poldistanz bestätigt meine Wahrnehmung, daß die mittlere Intensität der Streifen I9 I3-I 4 stetig vom Nordpol gegen den Südpol abnahm, wobei eine scheinbare zeitliche Entwicklung des Streifensystems vorgetäuscht wird, die für Untersuchungen über die Entwicklung der »Kanäle» geradezu verhängnisvoll sein muß.

Hörsholm, I 9 I 7 Juli.

H. E. Lau. so ist die Reduktion wegen der Instrumentalfehler

$$
=\mathcal{F} i+C c+A a
$$

wobei $\mathcal{F}==(\operatorname{ctg} \varphi \pm \operatorname{tg} \delta), C= \pm \sec \delta, A=( \pm \operatorname{tg} \delta-\operatorname{tg} \varphi)$ ist. $i$ berechnet man am besten direkt aus den Ablesungen am Niveau, indem man den mit $\sin \varphi$ multiplizierten Parswert anwendet. Diese neuen Größen $i$ und $a$ sind einfach die Projektionen der alten auf den durch den Westpunkt gehenden Stundenkreis, positiv, wenn sie nach Norden liegen. Die praktische Berechnung geschieht so:

$$
\begin{aligned}
& \operatorname{ctg} \varphi=\cdots \cdots \quad \mathcal{F}=\operatorname{ctg} \varphi+\operatorname{tg} \delta \\
& \operatorname{tg} \delta=\cdots \cdots \quad C=\sec \delta \\
& -\operatorname{tg} \varphi=\cdots \cdots \quad A=\operatorname{tg} \delta-\operatorname{tg} \varphi .
\end{aligned}
$$

Dies gilt für obere Kulminationen; für untere kehrt man das Vorzeichen von $\operatorname{tg} \delta$ und $\sec \delta$ um. Man hat hierbei auch die Koeffizienten in der Reihenfolge, wie sie bei der Reduktion gebraucht werden. Diese Formeln sind allgemein gültig für beide Halbkugeln.

Triest, I 9 I 7 Aug. 2 I

\section{$1917 \mathrm{CP}$.}

Aus den Beobachtungen Sept. 14 Königstuhl und Sept. 23 Wien und einer neueren Beobachtung Dr. Palisas vom 3. Oktober habe ich folgende elliptische Bahnelemente und Ephemeride des Wolfschen in der Nähe des Ortes des Enckeschen Kometen gefundenen Planeten berechnet.

$$
\begin{aligned}
& \text { Ep. } 1917 \text { Okt. } 3.5 \mathrm{~m} \text {. Z. Greenw. } \\
& M_{0}=29^{\circ} 5^{\prime} 6 \\
& \omega=3944.2
\end{aligned}
$$

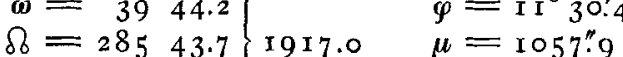

$$
\begin{aligned}
& i=443.7 \quad \log a=0.35^{\circ} 3^{8}
\end{aligned}
$$

Ephemeride für $\mathrm{I}^{\mathrm{h}} \mathrm{m}$. Z. Greenwich.

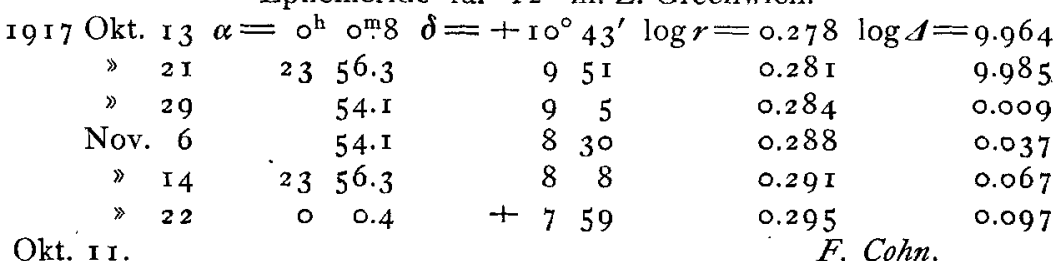

Kgl. Astronomisches Recheninstitut, I 9 I 7 Okt. I I.

Notiz. Mit dieser Nummer kommt für die Bezugsberechtigten Nr. 29 des Literarischen Beiblatts zur Versendung.

In halt zu Nr. 4906. A. Wilkens, Methoden zur Ermittelung der speziellen und der absoluten Koordinatenstörungen der Planeten der Jupitergruppe durch Jupiter. 145. - Neue Sterne in M roo = NGC 4321. 155. - H. E. Lau. Saturn und sein Ring. 157. - H. E. Lau. Über die Sichtbarkeit der Marskanäle in der Opposition 1913-14. 159. $-F$. R. Zueck. Zur Reduktion der Beobachtungen am Passagenrohr. 159. - 1917 CP. I 59. - Notiz. 159 . 